\title{
Variability in airway inflammation sampled by bronchoalveolar lavage
}

\section{To the Editor:}

The study by WARD et al. [1], designed to examine the variability in airway inflammation sampled by bronchoalveolar lavage in stable asthmatics, is a worthwhile and commendable piece of research. However, given the variability in the methacholine responsiveness found on the two separate study days, there are some issues with regard to the data analysis. Previous workers have suggested that greater than a plus or minus twofold change in airway responsiveness could be regarded as significant [2]. If one accepts that, in the study by WARD et al. [1], a greater than fourfold difference in the provocative dose of methacholine causing a $20 \%$ decrease in forced expiratory volume in one second (PD20) between the two study days can be considered as significantly different, then five patients should have been excluded from their analysis, i.e. patients No. 6, 12, 13, 15 and 20. These subjects had a variation in their PD20 of 5.3 to 125 fold between the two study days. If this subgroup were removed, and they could have been removed prospectively if the criteria for similar asthmatic status had included PD20 variation, what effect is there on the conclusions of the study?

Determinants of asthma stability relate to symptoms, medications, airway function, and also airway responsiveness. Limits for the comparison could be set for each of these items. The study [1] illustrates the challenge that we have in accepting absolute numbers that have been measured, without considering the errors that can occur. The team from Melbourne should be congratulated on their efforts to provide more evidence for the variability of measurements.

\section{References}

1. Ward C, Gardiner PV, Booth H, Walters EH. Intrasubject variability in airway inflammation sampled by bronchoalveolar lavage in stable asthmatics. Eur Respir $J$ 1995; 8: 1866-1871.

2. Ryan G, Dolovich MB, Obminski G, et al. Standardization of inhalation provocation tests: influence of nebulizer output, particle size, and method of inhalation. J Allergy Clin Immunol 1981; 67: 156-161.

\section{R.E. Ruffin}

Thoracic medicine, The Queen Elizabeth Hospital, 28 Woodville Road, Woodville SA 5011, Australia.

\section{REPLY}

From the authors:

Thank you for inviting a response to the letter by Dr Ruffin, which we feel raises some interesting issues regarding our recent publication. The study represented an attempt to examine the intrasubject variability of cellular and solute parameters in repeated $180 \mathrm{~mL}$ bronchoalveolar lavage (BAL) procedures in 20 clinically stable but symptomatic asthmatics. By "stable" we meant that they had no exacerbations or change in medication between procedures or for a reasonable time before. We subsequently formulated sample size estimates for pharmacological intervention studies involving repeated BAL; information which is lacking in the literature. Patients were selected on the basis that they represented the sort of subjects most likely to be involved in prospective studies of the effects of asthma therapy on airway pathology. Hence, they were young, with reasonably well-preserved lung function, on little or no inhaled corticosteroid, with quite marked bronchial hyperresponsiveness and variability in lung function. They would be chosen for a therapeutic intervention study largely on these criteria, so that it would be ethical to increase or change their medication, and it would be reasonable to expect that a significant improvement could be obtained both in clinical and bronchoscopic end-points.

The variability that we documented was, therefore, a product of biological variation in parameters, combined with the variability inherent in performing duplicate BAL analyses over time. It would seem intuitive, as is pointed out in the correspondence, that the inclusion of subjects with variable lung function and bronchial responsiveness to methacholine might influence the findings of our study. We avoided performing too many subanalyses on the original data set, but it does seem very reasonable to follow Dr Ruffins suggestion. In table 1 we present the original standard deviation of the differences for five salient indices measured in the paper, together with values following exclusion of the individuals with the most marked variability in lung function (patients Nos. 6, 12, 13, 15 and 20 in the original paper). It should be noted that, in the estimation of 95\% limits of agreement for the PD20 measurements reported in the paper, subject No. 15 was excluded, on the basis that this represented a clear outlier.

Table 1. - Relationship between the original standard deviation of the differences for five indices measured and values following exclusion of the five individuals (patients Nos. 6, 12, 13, 15 and 20) with the greatest variability in lung function

\begin{tabular}{lcc}
\hline Parameter & $\begin{array}{c}\text { SD original } \\
\text { data }\end{array}$ & $\begin{array}{c}\text { SD excluding 5 } \\
\text { subjects }\end{array}$ \\
\hline Total cell count $\times 10^{3} \cdot \mathrm{mL}^{-1}$ & 78 & 74 \\
Macrophages \% & 16 & 18 \\
Lymphocytes \% & 13 & 14 \\
Eosinophils \% & 1 & 1 \\
Albumin $\mu \mathrm{g} \cdot \mathrm{mL}^{-1}$ & 40 & 35 \\
\hline
\end{tabular}


This approach was suggested by BLAND and Altman [1]. On exclusion of the five most variable subjects, 95\% limits of agreement for PD20 measurements, expressed as a ratio, changed from $0.08-13$ (as quoted in the paper) to $0.3-7$. Hence, in the original analysis for $95 \%$ of subjects a second PD20 measurement would be expected to be 0.08-13 times the first; whereas, for the second analysis we would expect $95 \%$ of subjects to have a second PD20 0.3-7 times the first (about 2.5 doubling doses).

The standard deviation of the differences for repeated measurements, quoted in table 1, represents a useful summary statistic for documenting variability [1], and was used in the sample size calculations in our study. Table 1 shows that the SD of the differences were very similar when the original data were compared with an analysis excluding the subjects with the most variable physiology. In addition, plots of change in inflammatory indices against changes in log PD20 indicated that there were no relationships between these parameters. The findings of our study, therefore, remain essentially unaltered after considering this subanalysis.

In conclusion, we would like to point out that the purpose of our study, along with a recently published paper relating to airway inflammation in biopsies [2], was not to didactically construct absolute numbers for study design but was, rather, an attempt to contribute to a debate about methodology, that we feel is important. Our experience is that, in studies of "mild-to-moderate" symptomatic asthmatics, using the bronchoscopic end-points of BAL and endobronchial biopsy, about 15 patients is probably an optimal number.

\section{References}

1. Bland JM, Altman DG. Statistical methods for assessing agreement between two methods of clinical measurement. Lancet 1986; i: 307-317.

2. Richmond I, Booth $\mathrm{H}$, Ward C, Walters EH. Intrasubject variability in airway inflammation in biopsies in mildto-moderate stable asthma. Am J Respir Crit Care Med 1996; 153: 899-903.

\section{Ward, E.H. Walters}

Dept of Respiratory Medicine, Alfred Hospital and Monash University Medical School, Prahran, Melbourne, Victoria, Australia 3181. 